\title{
A comperative clinical study of mucotomy and KTP laser treatment of the inferior turbinate in allergic and non-allergic subjects
}

\author{
Ágnes Patzkó*, Eszter Tóth, Krisztina Somogyvári, Imre Gerlinger \\ Department of Otorhinolaryngology, Head and Neck Surgery, University Medical School of Pécs, Hungary;*Corresponding Author: \\ apatzko@yahoo.com
}

Received 20 July 2010; revised 20 August 2010; accepted 25 August 2010.

\begin{abstract}
Mucotomy is one of the most frequently applied surgical techniques for the management of inferior turbinate hyperplasia. Mucotomy guarantees patent airway, however, it might lead to the emergence of sicca syndrome. In contrast, KTP (potassium titanyl phosphate) laser treatment spares the medial part of the inferior turbinate mucosa, contributing to maintenance of physiological nasal function. A retrospective comparative clinical study was performed to reveal the advantages and side-effects of both surgical methods in medium- and long-term in allergic rhinitis and non-allergic patients. Furthermore, we wished to determine the exact indications of the up-to-date laser treatment. Ninety-one of the 117 patients who underwent bilateral turbinate surgery during an 8-year period (2000-2007) responded to our questionnaire focusing on subjective postoperative changes. Patients were separated into 6 groups, based on the type of operation they underwent, the length of the follow-up and whether they suffered from allergies. The major complaint, nasal obstruction, improved in all 6 groups, which reached significance $(p<0.05)$ in 4 groups. The most pronounced improvement was observed in the group of non-allergic patients with medium-term follow-up who underwent mucotomy. Nevertheless, a serious sideeffect: crusting also increased significantly $(p<$ 0.05 ) in the latter group, while it was absent in allergic patients with medium-term follow-up, who underwent mucotomy. These results lead us to propose the following protocol for the treatment of inferior turbinate hyperplasia: 1) after unsuccessful conservative treatment, laser treatment is suggested for non-allergic patients;
\end{abstract}

2) following unsuccessful conservative and even repeated laser treatment in the allergic group, mucotomy or turbinoplasty should be attempted.

Keywords: Crusting; Inferior Turbinate; KTP Laser; Mucotomy; Turbinoplasty

\section{INTRODUCTION}

Complete or incomplete inferior turbinate resection procedures are commonly used surgical techniques worldwide in both children and adults, in out- or in-patient settings. Most surgeons agree with the logical, but potentially misleading idea that the patients' complaints, especially nasal obstruction will be improved if nasal airflow is increased [1]. The most important factor determining airway resistance is the nasal valve. The nasal resistance is additionally influenced by the nasal cycle, which involves the alternating congestion and decongestion of the septal and inferior turbinate veins every 4-6 hours. As the inferior turbinate shrinks, this contributes to an increased nasal valve area, a decreased nasal resistance and an improved airflow [2].

There is considerable controversy in the literature concerning the management of inferior turbinate enlargement. The origins of this debate date back more than a hundred years, when the routine procedure was total turbinectomy $[3,4]$, which was accompanied by the adverse effects of serious bleeding, atrophic rhinitis and crusting [5]. Subsequently, various types of partial turbinectomy gained popularity [6-9]. Some of the recent surgical approaches, such as diathermy or cryosurgery cause tissue necrosis, and the subsequent fibrosis has been claimed to result in inferior turbinate reduction $[10,11]$. Nevertheless, disappointing outcome was reported in long-term follow-up patients, who underwent electrocauterization [7], as well as in the case of total inferior turbinate resection, indicating that the side-effects 
have been underestimated [12]. Treatment options utilizing the most up-to date technology (lasers and radiofrequency devices) are currently offered, though their outcomes have still not been adequately analysed [13-16].

Subjective patient complaints do not necessarily correlate with the objectively measured decrease in postoperative nasal resistance (determined by rhinomanometry, acoustic rhinometry) as an indication of excellent result of surgery [17-19]. Our paper assesses the outcome of mucotomy and KTP laser treatment of the inferior turbinate in both medium- and long term in allergic and non-allergic patients, with the aims of identifying the advantages and side-effects of these techniques from the aspect of quality of life of the patients, and of determining exact indications in order to elaborate a therapeutic protocol.

\section{PATIENTS AND METHODS}

\subsection{Patients}

A retrospective analysis of 117 patients who underwent bilateral turbinate surgery during an 8 year period (2000-2007) was planned. In 97 subjects (83\%) mucotomy and in 20 cases (17\%) laser treatment of the inferior turbinates had been carried out. Ninety- one (39 female, 52 male; mean age at surgery $37 \pm 13,7$ years, range: $14-73$ years; 74 mucotomy, 17 KTP laser-treated) of them (78\%) responded to a questionnaire focusing on subjective postoperative changes (Appendix). Patients could respond with a yes, no or not sure to all questions. For the analysis, the patients were divided into 6 groups, based on the type of operation they had undergone, the length of the follow-up (medium-term or long-term) and whether they suffered from allergies (Table 1).

The indication of bilateral mucotomy or laser treatment was based on the detailed medical history and physical examination (anterior rhinoscopy and nasal endoscopy). Objective measurements (rhinomanometry and acoustic rhinometry) were not performed. Allergic rhinitis was verified by means of the Prick-test and blood test for specific IgE detection besides the typical symptoms (itchy eyes, sneezing, nasal discharge and blockage and headache). The most common causes of non-allergic turbinate hyperplasia were medicamentous rhinitis and vasomotor rhinitis. Sixty-five of the 91 patients underwent septoplasty at the same time as (63 patients, $97 \%$ ) or before the turbinate surgery ( 2 patients, $3 \%$ ), while 3 patients had a septal reoperation simultaneously with or before the turbinate surgery $(5 \%)$. In cases of suspected chronic rhinosinusitis, a CT scan and nasal endoscopy facilitated the establishment of the indication for surgery.

\subsection{Surgical Procedures}

Mucotomy: Following medialization of the turbinate, the hyperplastic mucosa was surgically removed using straight scissors. In cases of significant hypertrophy excessive bone was not preserved. Occasional severe bleeding from the sphenopalatine artery was treated with a nasal tamponade. If necessary, the enlarged posterior pole of the inferior turbinate was also resected (Figure 1).

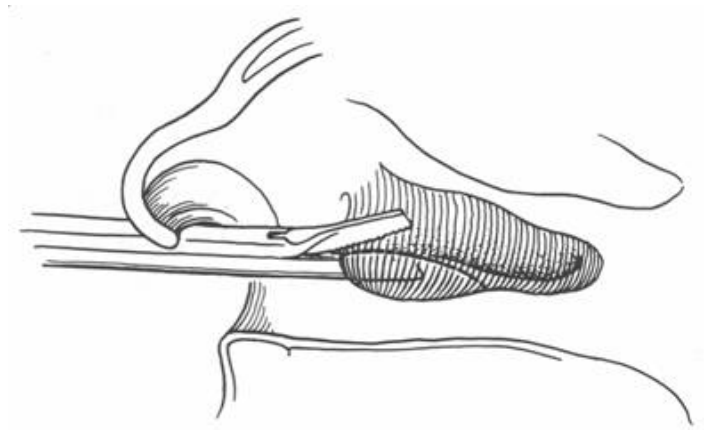

Figure 1. Schematic illustration of mucotomy.

Table 1. Groups of patients.

\begin{tabular}{lcc}
\hline \multicolumn{1}{c}{ Description } & Group abbreviation & No. of patients \\
\hline Medium-term follow-up of allergic patients who underwent mucotomy & M2A & 29 \\
Medium-term follow-up of non-allergic patients who underwent mucotomy & M2NA & 29 \\
Long-term follow-up of allergic patients who underwent mucotomy & M7.5A & 5 \\
$\begin{array}{l}\text { Long-term follow-up of non-allergic patients who underwent mucotomy } \\
\begin{array}{l}\text { Medium-term follow-up of allergic patients who underwent KTP laser } \\
\text { treatment }\end{array}\end{array}$ & KTP2A & 11 \\
$\begin{array}{l}\text { Medium-term follow-up of non-allergic patients who underwent KTP laser } \\
\text { treatment }\end{array}$ & KTP2NA & 10 \\
N.B: medium-term follow up: 2.11 years (range: 1.8-2.4 years) long-term follow up: 7.48 years (range: 7.1-7.9 years)
\end{tabular}


KTP laser treatment of the inferior turbinate: A KTP laser (wavelength: $532 \mathrm{~nm}$ ) has been applied for the treatment of our patients regularly since 2004. This type of laser combines ideal rhinological features of ablation, vaporization and coagulation. Furthermore, hemoglobin absorbs the laser light, providing a blood-free environment. The hand-piece allows the laser light beam to be directed towards the hidden parts of the nasal cavity (Figures 2(a) and 2(b)). In the course of the operation, the laser probe containing a $0.6 \mathrm{~mm}$ laser fiber was targeted to the posterior pole of the inferior turbinate under endoscopic control. The laser beam, applied at $10 \mathrm{~W}$ was conducted from the posterior to the anterior pole of the inferior turbinate in about 20-40 seconds, burning a trench into the surface of the inferior turbinate (Figure 3). In some cases, two parallel trenches were formed. In

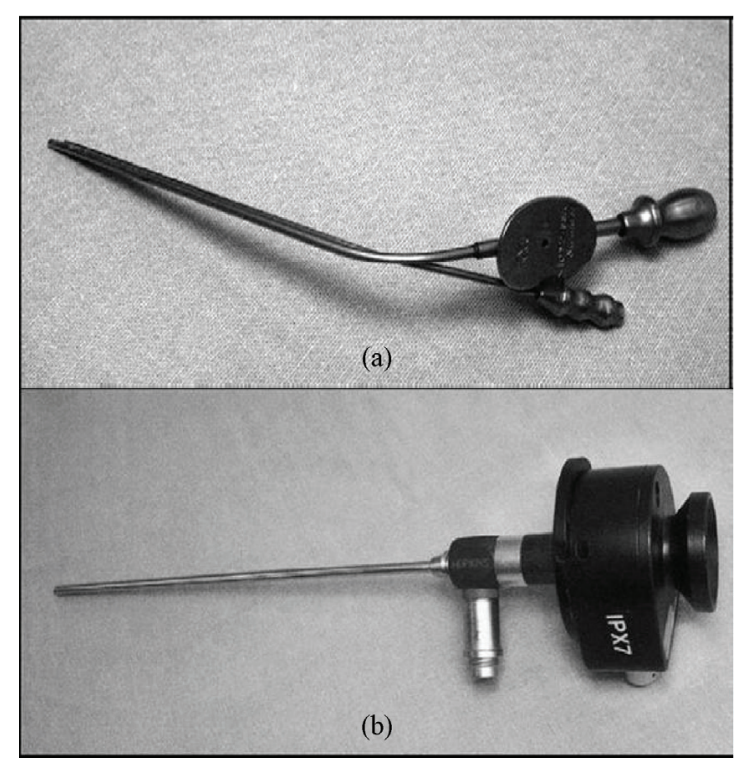

Figure 2. Fibre-optical hand piece with a built-in sucker (a). filter fixed to endoscope (b).

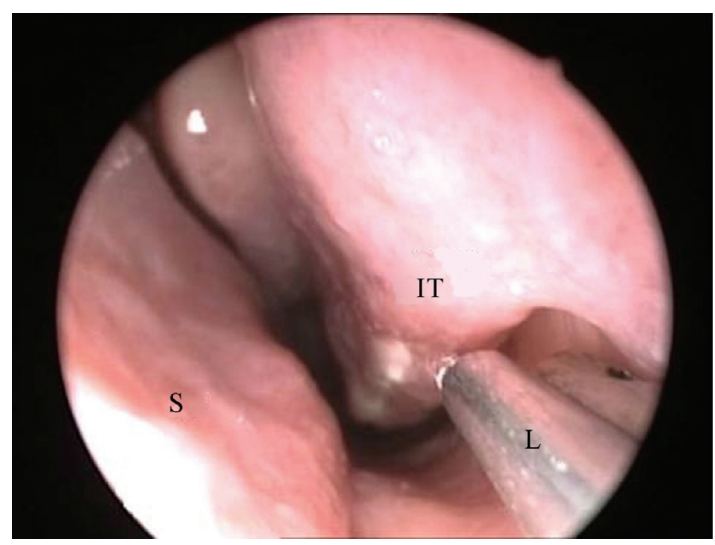

Figure 3. A trench was burnt into the surface of the inferior turbinate applying the laser in continuous contact mode at $10 \mathrm{~W}$. (S: septum, IT: inferior turbinate, L: laser fibre-optic). case of extreme anterior pole hyperplasia a brief additional laser treatment was applied to the submucosa. Special care was taken to spare the medial surface of the inferior turbinate. After laser treatment, there was no need for a tamponade.

\subsection{Statistical Analysis}

The changes reported by the patients between the preoperative and postoperative conditions were analyzed by means of the McNemar test. The answers relating to a given symptom were analyzed with the Wilcoxon signed rank test. We considered a probability level of $p<0.05$ as significant.

\section{RESULTS}

Our study focused on nasal breathing, crusting, smell detection and headache. Overall, the questions dealing with nasal breathing revealed a significant number of patients who no longer suffered from nasal obstruction in all the non-allergic groups and in group M2A (Figure 4). The most pronounced improvement occurred in group M2NA. Postoperatively, 23 of the 29 subjects (79\%) of this group regained the nasal respiratory function. Positive, though non-significant changes were observed in groups M7.5A and KTP2A. Whereas the latter two groups of allergic subjects reported difficulty with nasal breathing preoperatively, 3 of the 7 in group KTP2A and 3 of the 5 in group M7.5A experienced patent airways postoperatively.

The outcomes of the two surgical procedures were compared from the aspect of crusting. In group M2NA, the incidence of crusting was significantly higher postoperatively $(\mathrm{p}<0.05)$ : preoperatively $6(21 \%)$, postoperatively $16(55 \%)$ of the 29 subjects complained of crusting (Figure 5). We did not see such negative effects of the surgery on crusting in group M2A. All of these patients had undergone bilateral inferior turbinate surgery and consequently significantly more of them presented with bilateral crusting. Ten $(62 \%)$ of the 16 patients in group M2NA, who reported crusting experienced a thick, smelly discharge from the nasopharynx, 3 (19\%) complained of a watery nasal discharge, and only $3(19 \%)$ patients reported no nasal discharge. We did not find significant changes in crusting in the remaining 4 groups, regardless of the type of surgery.

When considering smell detection, there was a postoperative improvement in all 6 groups, though it did not reach the level of significance (Figure 6). The questions concerning headache, ear pain and reduced hearing acuity revealed significant differences post-operatively. Headache of the M2NA patients was alleviated. No patients developed headaches after the operation and 6 of 


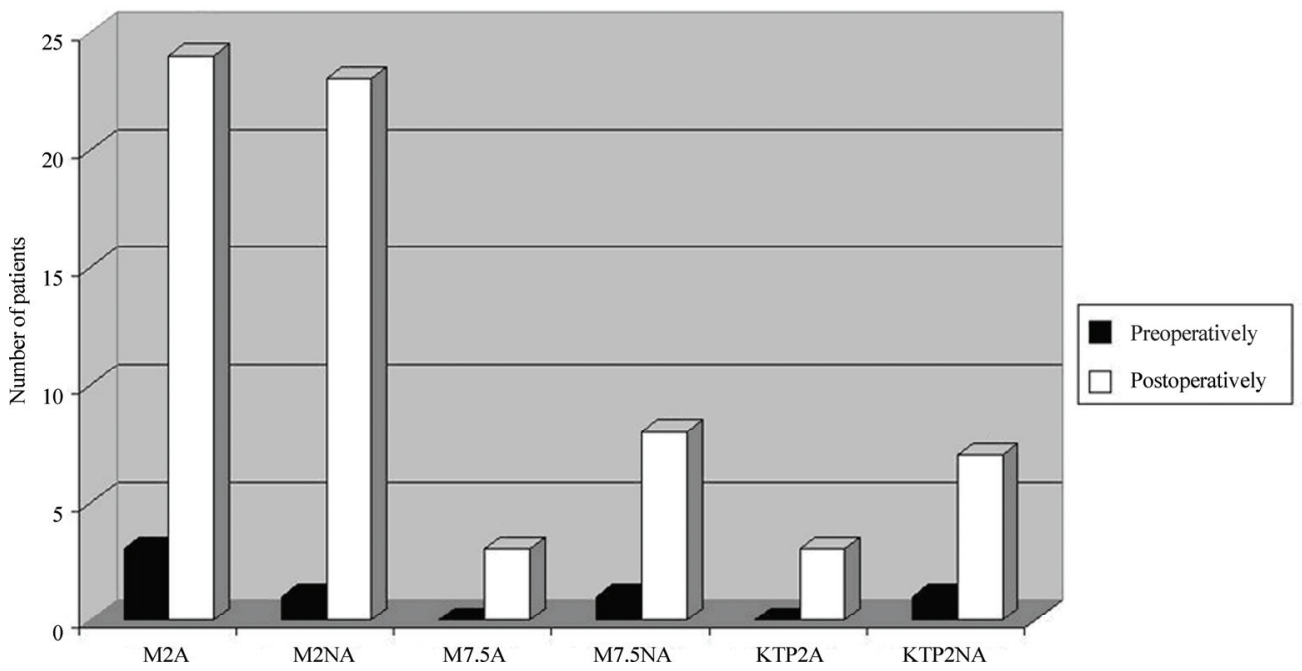

Figure 4. Improvement in nasal breathing in patients who underwent mucotomy or KTP laser treatment. (For nomenclature, see Table 1).

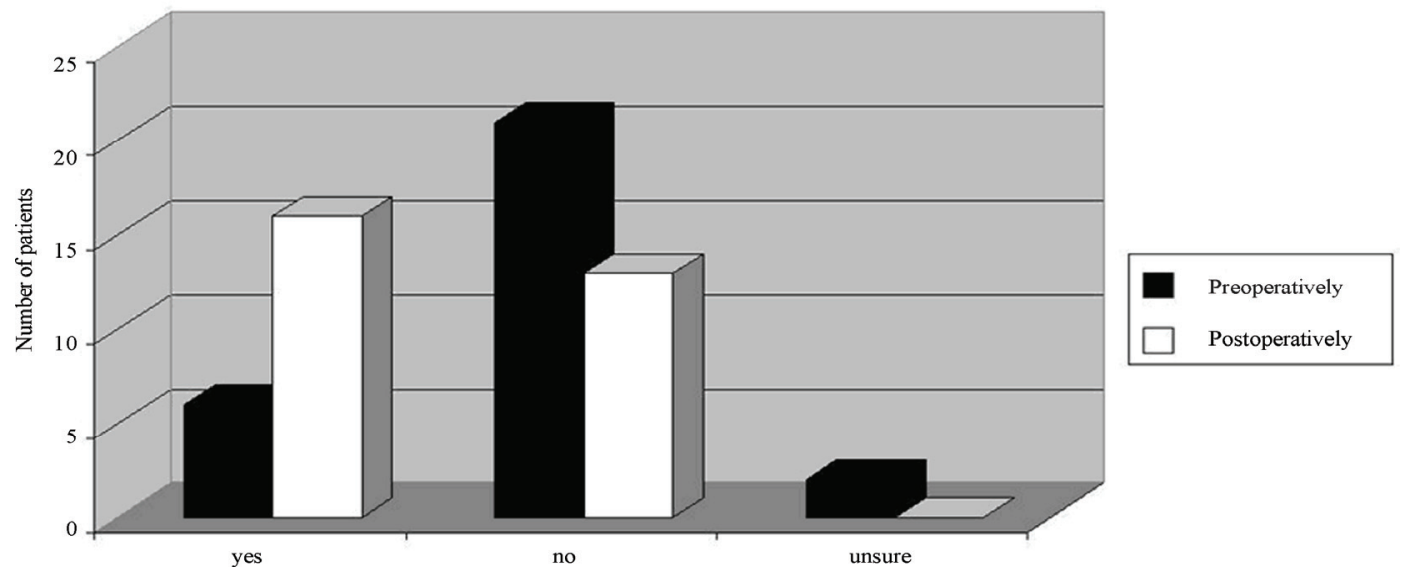

Figure 5. Changes in the incidence of crusting in group M2NA. These patients displayed the most marked improvement in nasal breathing besides the highest incidence of postoperative crusting.

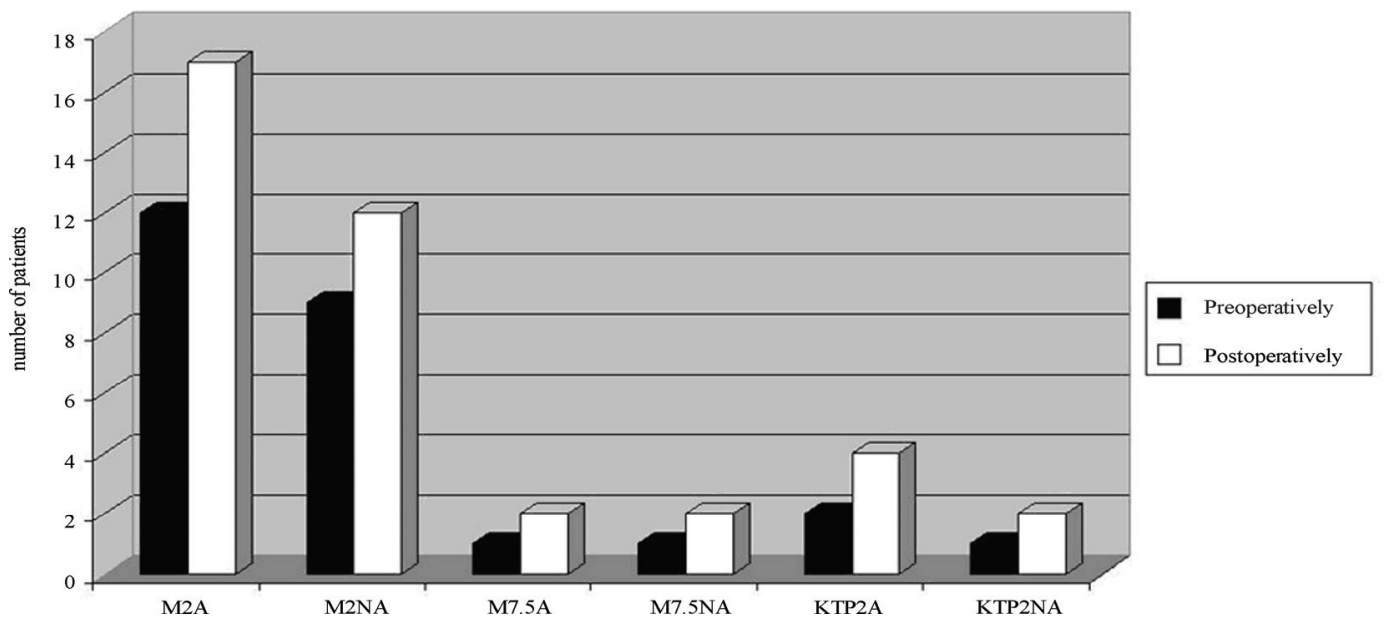

Figure 6. Moderate, non-significant improvement of sense of smell in all study groups. 
the 15 patients who suffered from headaches preoperatively became symptom-free. Moreover, among the M2A patients, the ear pain diminished and the hearing acuity improved postoperatively, with 6 of the 11 patients no longer having complaints.

\section{DISCUSSION}

Adequate treatment of inferior turbinate enlargement poses a challenge for functional nasal surgery. Unfortunately, detection of the underlying cause of the hyperplasia (allergy, medicamentous rhinitis, vasomotor rhinitis or compensatory hyperplasia caused by a septal deviation) and its conservative management do not always result in symptomatic relief. The large variety of surgical interventions reflects the lack of consensus (Table 2). We believed that a study comparing the subjective outcome following mucotomy or KTP laser treatment of the inferior turbinate would be helpful in developing a therapeutic protocol resulting in the best possible quality of life for the patient. Although rhinomanometry and acoustic rhinometry are mainly used for scientific purposes, in the future these procedures should be applied both preand postoperatively. Their use would allow a detailed analysis of the correlation between objective and subjective symptoms [17].

We emphasize that both mucotomy and KTP laser treatment significantly relieved the nasal obstruction, which was the major complaint of our patients. After the interventions, $71 \%$ of the subjects questioned, $56 \%$ of the total patient cohort reported a full restoration of their nasal respiratory function. In group M2NA, where the improvement of nasal obstruction was most pronounced, the incidence of postoperative crusting increased significantly. Most of these patients complained of a thick, smelly nasal discharge and required regular nasal lavage, complaints indicative of the emergence of atrophic rhinitis. Atrophic rhinitis and the consequent sicca syndrome are unpleasant consequences of mucotomy and cause a deterioration of the quality of life. Oezena was not observed among our patients. Fifty-five per cent of the questioned M2NA patients (43\% of the total patient cohort) developed crusting. Surprisingly, crusting was not reported to be considerably more frequent in the longterm follow-up groups who underwent mucotomy (M7.5A and M7.5NA). There may be several reasons that explain this finding: the patients might have become used to their symptoms and applied nasal ointment or paraffin oil to their nose, the allergic patients could have received medication, or mucosal regeneration could have occurred. Despite the lack of symptoms referring to atrophic rhinitis, we realize the importance of an even longer follow-up [12]. The M2A patients did not present with the negative features of atrophic rhinitis and the intervention did mitigate the nasal obstruction in these patients. In our opinion, the abundant secretion of the remaining nasal mucosa protected them against atrophic changes. In the future histological examination could confirm this hypothesis.

Table 2. Types of interventions on the inferior turbinate.

\begin{tabular}{|c|c|c|}
\hline Surgery & Advantages & Disadvantages \\
\hline \multicolumn{3}{|l|}{ Mechanical Intervention } \\
\hline Medialization of the Inferior Turbinate & $\begin{array}{l}\text { easy to perform, low risk, can be combined with } \\
\text { other techniques }\end{array}$ & $\begin{array}{l}\text { little symptomatic relief, mucosa remains } \\
\text { hyperplastic }\end{array}$ \\
\hline \multicolumn{3}{|l|}{ Destructive Interventions } \\
\hline Cryosurgery & $\begin{array}{l}\text { easy to perform, local an aesthesia is sufficient, no } \\
\text { need for tamponade }\end{array}$ & $\begin{array}{l}\text { symptomatic relief may occur several months } \\
\text { later, bleeding, adhesions, no long-term relief, } \\
\text { crusting }\end{array}$ \\
\hline Electrocautery & $\begin{array}{l}\text { local anesthesia, good haemostasis, relatively ef- } \\
\text { fective }\end{array}$ & $\begin{array}{l}\text { only short-term symptomatic relief (up to 1-2 } \\
\text { years)? crusting, adhesion, bleeding, infection }\end{array}$ \\
\hline Lasers & $\begin{array}{l}\text { local anesthesia, good haemostasis, relatively ef- } \\
\text { fective in medium-term ( } 2 \text { years })\end{array}$ & $\begin{array}{l}\text { adhesion, expensive equipment, training need- } \\
\text { ed }\end{array}$ \\
\hline Radiosurgery & $\begin{array}{l}\text { no adhesion, good hemostasis, relatively effective } \\
\text { in medium-term ( } 2 \text { years })\end{array}$ & high cost, limited experience \\
\hline \multicolumn{3}{|c|}{ (1) } \\
\hline Partial Turbinectomy & easy to perform, long-lasting results & crusting \\
\hline Total Turbinectomy & easy to perform & crusting, bleeding, tamponade \\
\hline Submocosal Resection & $\begin{array}{l}\text { preserves mucosal function, effective, prevents } \\
\text { atrophic rhinitis }\end{array}$ & $\begin{array}{l}\text { tamponade, frequent recurrence of symptoms, } \\
\text { persistent rhinorrhoea }\end{array}$ \\
\hline Vidian Neurectomy & $\begin{array}{l}\text { rhinorrhoea ceases, symptomatic } \\
\text { relief for years }\end{array}$ & $\begin{array}{l}\text { dry eyes; intermittent eye or facial pain; pala- } \\
\text { tal, dental and facial numbness }\end{array}$ \\
\hline
\end{tabular}


The number of M2NA patients suffering from headache was significantly lower postoperatively. All of the patients whose headache ceased had presented with a septal deviation and underwent simultaneous septal surgery. We suggest that the remission of the headache was due to the removal of the septal spine, which penetrated the inferior turbinate and resulted in ganglion sphenopalatine neuralgia. We do not regard the symptomatic relief as a consequence of the inferior turbinate surgery, but as a coincidence, for the percentage of patients who underwent simultaneous septal surgery was extremely high in this group (86\%). We observed that the preoperative ear pain, fullness sensation and decreased hearing acuity improved significantly in the M2A patients. These patients could have exhibited swelling of the mucosa in the upper respiratory tract, due to allergy, resulting in chronic inflammation of the Eustachian tube. The symptomatic relief was probably due to the appropriate treatment of allergies. Mucotomy markedly reduces the mucosal surface and may result in a mucociliary dysfunction increasing the susceptibility to infections. In spite of this, an increased infection rate was not seen in any of the groups. A rare, but severe complication of the treatment of inferior turbinate enlargement is a deterioration of visual acuity, linked to retrograde embolism or retinal vasospasm [20]. One of the mucotomy patients reported a permanent severe visual loss.

Our observations lead to suggest KTP laser therapy for non-allergic patients. We assume that submucosal scarring prevents the fast swelling and shrinkage of the mucosa. Furthermore, laser treatment alters the autonomous nervous system control, impacts on the nasal cycle and reduces the number and the function of the mucinous glands [21]. Two previous studies noted the limited success of the laser treatment of allergic patients as compared with non-allergic patients $[14,15]$. The reason for this outcome might be that after the operation the patients return to their usual allergen-contaminated environment, which results in chronic inflammation of the mucosa. It is suggested that these patients should be offered more radical treatment options. The small sample size allows us to draw only limited conclusions from our study. We consider that, as the first step, allergic patients should be offered laser treatment, since it is minimally invasive and can easily be adjusted to the patients' needs. It gives comparable or even better results than conventional inferior turbinate surgery. It can be performed at out-patient clinics and does not require the unpleasant tamponade of the nose. Janda et al. concluded that the different types of lasers used in nasal surgery can provide equally beneficial results if correctly set and applied [11]. The long-term effectiveness of laser treatment depends greatly on the level of postoperative care. We re- commend that patients should regularly perform nasal lavage in order to prevent synechiae formation, inflammation and atrophic rhinitis. However, it is indispensable to explore the possible long-term side-effects of laser treatment.

The primary advantage of more invasive procedures (turbinectomy, turbinoplasty and resection techniques) is the achievement of the long-term airway patency. Unfortunately, radical operations may trigger the appearance of the sicca syndrome. The best approach appears to be the stepwise treatment of inferior turbinate hyperplasia. Following unsuccessful conservative therapy, laser treatment should be offered to both allergic and nonallergic patients, though long-term benefits are appreciably less likely for allergic patients. In the event of unsuccessful repeated laser treatment, we suggest that patients should be selected for turbinoplasty. In the future, several other new treatment options (e.g. radiofrequency treatment) could be considered [16].

\section{REFERENES}

[1] Clement, W.A. and White, P.S. (2001) Trends in turbinate surgery literature: A 35-year review. Clinical Otolaryngology and Allied Sciences, 26(2), 124-128.

[2] Eccles, R. (2000) Nasal airflow in health and disease. Acta Otolaryngol, 120(5), 580-595.

[3] Holmes, C.R. (1900) Hypertrophy of the turbinated bodies. New York Medical Journal, 72, 529.

[4] Jones, T.C. (1895) Turbinotomy. Lancet, 2, 496.

[5] Jackson, L.E. and Koch, R.J. (1999) Controversies in the management of inferior turbinate hypertrophy: A comprehensive review. Plastic and Reconstructive Surgery, 103(1), 300-312.

[6] Courtiss, E.H., Goldwyn, R.M. and O’Brien, J.J. (1978) Resection of obstructing inferior nasal turbinates. Plastic and Reconstructive Surgery, 62(2), 249-257.

[7] Meredith, G.M. (1988) Surgical reduction of hypertrophied inferior turbinates: a comparison of electrofulguration and partial resection. Plastic and Reconstructive Surgery, 81(6), 891-899.

[8] Pollock, R.A. and Rohrich, R.J. (1984) Inferior turbinate surgery: an adjunct to successful treatment of nasal obstruction in 408 patients. Plastic and Reconstructive Surgery, 74(2), 227-236.

[9] Vona, I. and Bauer, M. (1996) Az orrlégzés javítása turbinoplastica anteriorral és az apertura piriformis tágításával. Fül-orr-gégegyógy (Hungarian), 42, 227-230.

[10] Farmer, S.E. and Eccles, R. (2007) Understanding submucosal electrosurgery for the treatment of nasal turbinate enlargement. The Journal of Laryngology \& Otology, 121(7), 615-622.

[11] Janda, P., Sroka, R., Baumgartner, R., Grevers, G. and Leunig, A. (2001) Laser treatment of hyperplastic inferior nasal turbinates: A review. Lasers in Surgery and Medicine, 28(5), 404-413.

[12] Moore, G.F., Freeman, T.J., Ogren, F.P. and Yonkers, A.J. 
(1985) Extended follow-up of total inferior turbinate resection for relief of chronic nasal obstruction. Laryngoscope, 95(9 Pt 1), 1095-1099.

[13] Back, L.J., Hytonen, M.L., Malmberg, H.O. and Ylikoski, J.S. (2002) Submucosal bipolar radiofrequency thermal ablation of inferior turbinates: A long-term follow-up with subjective and objective assessment. Laryngoscope, 112 (10), 1806-1812.

[14] Janda, P., Sroka, R., Tauber, S., Baumgartner, R., Grevers, G. and Leunig, A. (2000) Iode laser treatment of hyperplastic inferior nasal turbinates. Lasers in Surgery and Medicine, 27(2), 129-139.

[15] Leunig, A., Janda, P., Sroka, R., Baumgartner, R. and Grevers, G. (1999) Ho: YAG laser treatment of hyperplastic inferior nasal turbinates. Laryngoscope, 109(10), 1690-1695.

[16] Sapci, T., Usta, C., Evcimik, M.F., Bozkurt, Z., Aygun, E., Karavus, A. and Peker, M. (2007) Evaluation of radiofrequency thermal ablation results in inferior turbinate hypertrophies by magnetic resonance imaging. Laryngoscope, 117(4), 623-627.

[17] Hirschberg, A. (2002) Rhinomanometry: An update. Journal for Oto-Rhino-Laryngology and Its Related Specialties, 64(4), 263-267.

[18] Jones, A.S., Willatt, D.J. and Durham, L.M. (1989) Nasal airflow: Resistance and sensation. The Journal of Laryngology \& Otology, 103(10), 909-911.

[19] Panagou, P., Loukides, S., Tsipra, S., Syrigou, K., Anastasakis, C. and Kalogeropoulos, N. (1998) Evaluation of nasal patency: Comparison of patient and clinician assessments with rhinomanometry. Acta Otolaryngol, 118(6), 847-851.

[20] Mabry, R.L. (1981) Visual loss after intranasal corticosteroid injection. Incidence, causes, and prevention. Arch Otolaryngol, 107(8), 484-486.

[21] Gerlinger, I., Lujber, L., Jarai, T. and Pytel, J. (2003) KTP-532 laser-assisted endoscopic nasal sinus surgery. Clinical Otolaryngology and Allied Sciences, 28(2), 67-71.

\section{Appendix: Questionnaire}

After the questions in this questionnaire, you will find several possible answers. Please, circle the correct response!

\begin{tabular}{|c|c|c|c|c|}
\hline 1 & Did material run into your mouth from the back of your nose before the operation? & Yes & No & I am not sure \\
\hline 2 & If it did what was this material like? & Thick & Thin & Watery \\
\hline 3 & If it did, did it have an unpleasant taste? & Yes & No & I am not sure \\
\hline 5 & Does material run into your mouth from the back of your nose since the operation? & Yes & No & I am not sure \\
\hline 6 & If it does, what is this material like? & Thick & Thin & Watery \\
\hline 8 & If it does run into your mouth, what colour is it? & Yellow & Green & White \\
\hline 9 & Could you breathe at all through your nose before the operation? & Yes & No & I am not sure \\
\hline 10 & Can you breathe at all through your nose since the operation? & Yes & No & I am not sure \\
\hline 11 & Did you notice crusty material in your nose before the operation? & Yes & No & I am not sure \\
\hline 12 & Have you noticed crusty material in your nose since the operation? & Yes & No & I am not sure \\
\hline 13 & $\begin{array}{l}\text { Did your acquaintances ever mention an unpleasant smell coming from your nose } \\
\text { before the operation? }\end{array}$ & Yes & No & I am not sure \\
\hline 14 & $\begin{array}{l}\text { Have your acquaintances ever mentioned an unpleasant smell coming from your nose } \\
\text { since the operation? }\end{array}$ & Yes & No & I am not sure \\
\hline 15 & Did you notice a change in your sense of smell before the operation? & Yes & No & I am not sure \\
\hline 16 & Have you noticed a change in your sense of smell since the operation? & Yes & No & I am not sure \\
\hline 17 & Did you often have headache before the operation? & Yes & No & I am not sure \\
\hline 18 & Do you often have headache since the operation? & Yes & No & I am not sure \\
\hline 19 & Did you have blurred vision before the operation? & Yes & No & I am not sure \\
\hline 20 & Have you had blurred vision since the operation? & Yes & No & I am not sure \\
\hline 21 & Did you notice any hearing loss or ear pain before the operation? & Yes & No & I am not sure \\
\hline 22 & Have you noticed any hearing loss or ear pain since the operation? & Yes & No & I am not sure \\
\hline
\end{tabular}

